\title{
Partially Hyperbolic Dynamics and 3-Manifold Topology
}

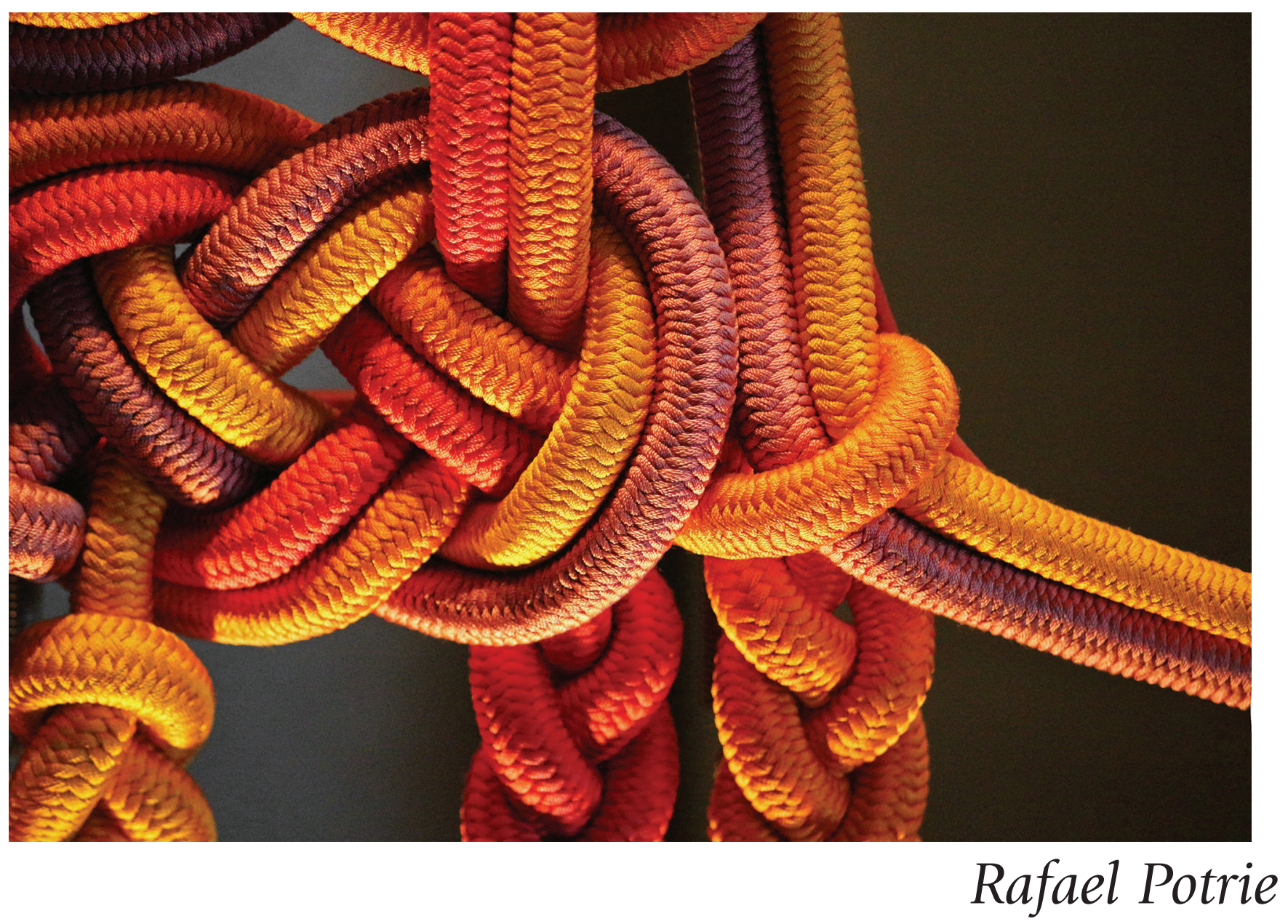

\section{Introduction}

The hairy ball theorem implies that the 2-dimensional sphere cannot admit a vector field without singularities. This is just an example of a restriction imposed by the topology of a phase space on the possible dynamics it can support. In this note we would like to present and relate two results in this direction. These are about restrictions imposed by the topology of certain 3-manifolds on the dynamics it can support.

Rafael Potrie is an associate professor at Universidad de la República, Uruguay. His email address is rpotri e@cmat. edu. uy.

For permission to reprint this article, please contact:

reprint-permission@ams.org.

DOI: https://doi.org/10.1090/noti2286
The first result we will present corresponds to the theory of Anosov flows and was proved by Margulis [Mar] when he was still an undergraduate student. It appears in an appendix to a paper of Anosov and Sinai [AnS]. The result was later revisited by Plante and Thurston [PT] who extended its scope and proposed a different approach that used some finer properties of foliations.

A flow $\phi_{t}: M \rightarrow M$ generated by a (smooth) vector field $X$ on a closed manifold $M$ is said to be an Anosov flow if there is a continuous $D \phi_{t}$-invariant splitting of the tangent bundle $T M=E^{s} \oplus \mathbb{R} X \oplus E^{u}$ satisfying that there is $t_{0}>$ 0 so that for every $v^{\sigma} \in E^{\sigma}(\sigma=s, u)$ a unit vector we have that $\left\|D \phi_{t_{0}} v^{s}\right\|<1<\left\|D \phi_{t_{0}} v^{u}\right\|$. This immediately implies that stable vectors (i.e., those in $E^{S}$ ) are contracted exponentially by $D \phi_{t}$ while unstable vectors (i.e., those in $\left.E^{u}\right)$ are expanded exponentially fast by $D \phi_{t}$. 


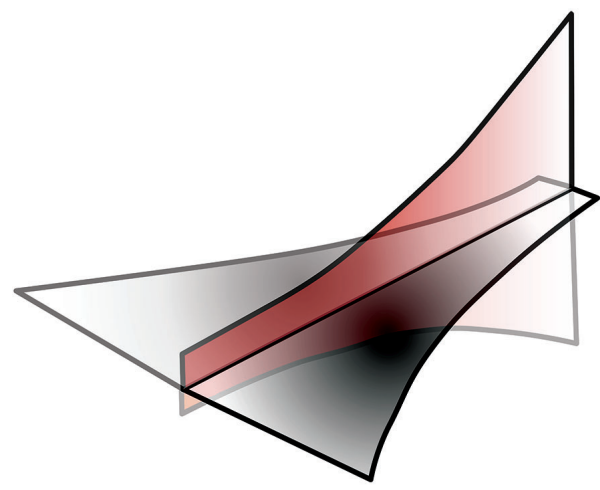

Figure 1. The local aspect of orbits of Anosov flow.

Examples of Anosov flows include geodesic flows in negative curvature [Ano] as well as suspensions of certain toral automorphisms. Their definition goes back at least to the paper of Anosov and Sinai [AnS] where they extracted the properties from geodesic flows in negative curvature needed to obtain ergodicity. We point out that in 3-manifolds we know that Anosov flows contain the space of robustly transitive flows (i.e., flows so that every perturbation has some dense orbit); see [Do, BDV]. The result by Margulis and Plante-Thurston says that if a 3-manifold admits an Anosov flow, then its fundamental group has exponential growth (see Theorem 3.1) and implies in particular that 3-manifolds such as the sphere $S^{3}$ or the 3-torus $\mathbb{T}^{3}=\mathbb{R}^{3} / \mathbb{Z}^{3}$ do not admit such flows.

The second result is more recent and essentially due to Burago and Ivanov [BI]. This result gives some obstructions for some mapping classes of certain 3-manifolds to admit partially hyperbolic diffeomorphisms. A diffeomorphism $f: M \rightarrow M$ is said to be partially hyperbolic if the tangent space $T M$ splits as a direct sum of non-trivial continuous subbundles $E^{s} \oplus E^{c} \oplus E^{u}=T M$ which are $D f$ invariant and satisfy that there is some $\ell>1$ so that for every $x \in M$, if $v^{\sigma} \in E^{\sigma}(x)(\sigma=s, c, u)$ are unit vectors, then

$$
\begin{aligned}
\left\|D f^{\ell} v^{s}\right\|<\min \left\{1,\left\|D f^{\ell} v^{c}\right\|\right\} & \text { and } \\
\left\|D f^{\ell} v^{u}\right\| & >\max \left\{1,\left\|D f^{\ell} v^{c}\right\|\right\} .
\end{aligned}
$$

Naturally, time one maps of Anosov flows (i.e., the diffeomorphism $f(x)=\phi_{1}(x)$ where $\phi_{t}$ is an Anosov flow) are examples where $E^{c}=\mathbb{R} X$. Many homogeneous dynamics (namely, those which have positive entropy) are partially hyperbolic. Some of them are not time one maps of Anosov flows; for instance, the action of a matrix $A \in$ $\mathrm{SL}(3, \mathbb{Z})$ in the 3 -torus $\mathbb{T}^{3}$ with three different real eigenvalues is such an example. Being partially hyperbolic is an open property in the $C^{1}$-topology, so it is possible to make $C^{1}$-small perturbations to the mentioned examples to obtain new examples.
In dimension 3, we will explain how the result of Burago-Ivanov implies that if a diffeomorphism $f: M \rightarrow$ $M$ is partially hyperbolic and the manifold does not have fundamental group of exponential growth, then $f$ cannot be homotopic to the identity. See Theorem 5.4 for a precise statement.

The connection between these two results will allow us to briefly comment on the classification of partially hyperbolic diffeomorphisms in 3-manifolds, referring the interested reader to recent surveys such as [CHHU, HP, Pot, BFFP, BFP] for a more complete presentation.

\section{Anosov Flows and Foliations}

Consider an Anosov flow $\phi_{t}: M \rightarrow M$ in a closed manifold $M$. The definition requires that the differential of the flow preserves some geometric structure. It could seem hard to check that a flow is Anosov, but it is important to remark that the existence of the $D \phi_{t}$-invariant bundles follows from the existence of a way more flexible structure, namely that of invariant cone-fields, which we will not define but just point out that these are objects that are robust (i.e., if a system has invariant cone-fields, then this is true in a $C^{1}$-open neighborhood) and somewhat easy to check (i.e., a computer can check whether a system is Anosov).

The importance of this infinitesimal condition is that it can be pushed into the manifold in a way that one obtains objects whose dynamics mimic the dynamics of the differential map.

Theorem 2.1 (Stable manifold theorem). The bundles $E^{S}$ and $E^{s} \oplus \mathbb{R} X$ are uniquely integrable.

We need to say a few words to explain what we mean. Let $E \subset T M$ be a $k$-dimensional subbundle of the tangent bundle of $M$. We say that $E$ is uniquely integrable if through every point $x \in M$, there is a $k$-dimensional submanifold $S_{x}$ everywhere tangent to $E$ such that every curve tangent to $E$ through $x$ is completely contained in $S_{x}$.

The same is true for $E^{u}$ and $\mathbb{R} X \oplus E^{u}$ by applying the theorem to $\phi_{-t}$. Notice that even when $\operatorname{dim}\left(E^{s}\right)=1$, showing its unique integrability is not obvious since these bundles are typically no better than Hölder continuous; one needs to appeal to dynamics to get this kind of result.

The proof of this result when $\operatorname{dim}\left(E^{s}\right)=1$ is not complicated and we will now sketch it. Assume for the sake of contradiction that there are two different curves $\gamma_{1}$ and $\gamma_{2}$ everywhere tangent to $E^{S}$ which separate at a point $x \in M$. Note that by considering $\phi_{t}$ with large $t$ we get that the curves $\gamma_{1}$ and $\gamma_{2}$ decrease their length exponentially fast. However, their transverse distance, measured along the direction $\mathbb{R} X \oplus E^{u}$, cannot decrease (see Figure 1), which provides a contradiction. To show unique integrability of $E^{S} \oplus \mathbb{R} X$ one just needs to flow the integral curves of $E^{s}$ by the flow (whose defining vector field is smooth, so uniquely integrable). 

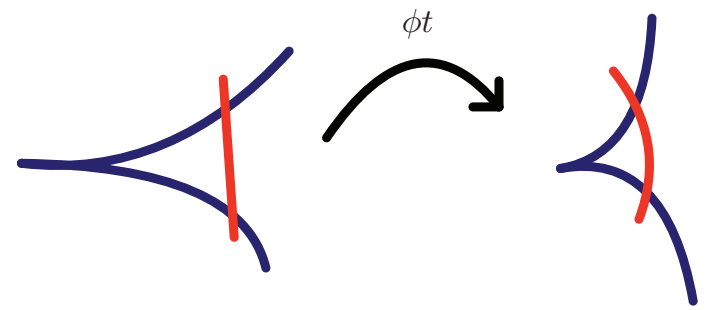

Figure 2. Flowing forward different curves tangent to $E^{S}$ through a given point gives a contradiction.

The curves tangent to $E^{S}$ and the surfaces tangent to $E^{s} \oplus \mathbb{R} X$ form what we call the strong stable and weak stable foliations, which together with their dual strong unstable and weak unstable foliations are one of the main tools to understand the dynamics and geometry of Anosov systems. Let us just state an easy fact about these that we will use later:

Proposition 2.2. There are no closed submanifolds $N$ of $M$ tangent to $E^{s} \oplus \mathbb{R} X$.

This is a direct consequence of the fact that the time $t_{0}$ map $\phi_{t_{0}}$ of the flow would be a diffeomorphism of the compact manifold $N$ whose derivative is everywhere contracting volume, which is impossible.

Recall that a foliation by surfaces of a 3-manifold $M$ is a partition of $M$ by injectively immersed $C^{1}$-surfaces (called leaves) that locally look like horizontal planes $\mathbb{R}^{2} \times\{t\}$ sitting inside $\mathbb{R}^{3}$ (i.e., there are charts sending leaves to horizontal planes; see Figure 3). An example of a foliation by surfaces would be the weak stable foliation of an Anosov flow in a 3-manifold.

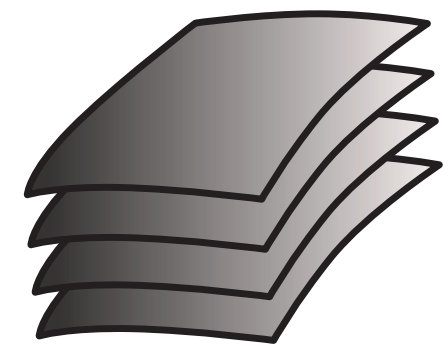

Figure 3. A foliation locally looks as a stack of surfaces.

A transversal to a foliation is an embedded circle which is everywhere transverse to the leaves of $\mathcal{F}$. Notice that if $M$ is compact, there are always transversals since a transverse curve intersecting a foliation box twice can be closed into a transversal. See Figure 4.

Theorem 2.3 (Novikov). Let $\mathcal{F}$ be a foliation by surfaces in a closed 3-manifold. Assume that there is a transversal $\gamma$ to $\mathcal{F}$ which is homotopically trivial. Then $\mathcal{F}$ has a closed leaf.

We will not prove this beautiful result which has several expositions. In fact, Novikov's result is much stronger and implies the existence of what are known as Reeb components. One should think that in 3-manifolds compact leaves (or Reeb components) of foliations play the role that singularities play in vector fields in surfaces, and therefore Novikov's theorem acts as the Poincaré-Bendixon's theorem in this setting. ${ }^{1}$ We refer the reader to [Ca, Chapter 4] for a friendly account of foliations in 3-manifolds.

\section{Margulis/Plante-Thurston"s Result}

In the late 60s Margulis showed the following beautiful result:

Theorem 3.1. Let $M$ be a closed 3-dimensional manifold admitting an Anosov flow $\phi_{t}$; then, the fundamental group of $M$ grows exponentially.

A finitely generated group $\Gamma$ has exponential growth if for some finite generating set $F \subset \Gamma$ it follows that the number of different group elements that can be written as a product of at most $n$ elements of $F \cup F^{-1}$ grows exponentially with $n$. This is independent of the finite generating set $F$.

In a closed manifold $M$ the fundamental group has exponential growth if and only if the volume of a ball or radius $R$ in $\widetilde{M}$, the universal cover of $M$, grows exponentially with respect to $R$. This means that if $\pi: \widetilde{M} \rightarrow M$ is the universal covering map, and we consider the metric induced by $\pi$ in $\widetilde{M}$, then there is a point $x \in \widetilde{M}$ and constants $c, \delta>0$ so that

$$
\operatorname{vol}(B(x, R))>c e^{\delta R},
$$

where $B(x, R)$ denotes the ball of center $x$ and radius $R$ in $\widetilde{M}$. To see the equivalence one just needs to find a compact fundamental domain in the universal cover and note that its volume must be finite; this way one can cover the ball of radius $R$ by deck transformations of bounded size and compare the growth of the volume of the ball with the growth of the fundamental group as a finitely generated group. This is the definition we will use to prove Theorem 3.1 .

It is an easy exercise to show that, up to changing the constants $c, \delta$, the definition is independent of the point $x \in \widetilde{M}$ as well as of the metric one pulls back from $M$. Thus this is indeed a topological property of $M$ which in fact only depends on its fundamental group. Under this assumption we say that $M$ has exponential growth of fundamental group.

\footnotetext{
${ }^{1}$ Even if much deeper, there is a part of the proof of Novikov's theorem (which is indeed enough to rule out homotopically trivial transversal loops for Anosov flows) that is very much modelled in the proof of Poincaré-Bendixon's theorem. It is known as Haefliger's argument: using the transverse loop, one constructs a disk whose boundary is transverse to the foliation and which is in general position; studying the induced flow on the disk is enough to find a configuration which is not compatible with Anosov flows, and such that with much more work produces a Reeb component. We note that for the partially hyperbolic case to be treated later, the full version of Novikov's theorem is important.
} 
The proof by Margulis [Mar] is direct and independent of any deep result in foliation theory (even if the foliations are used crucially). Later, Plante and Thurston [PT] gave a more conceptual proof that works for general codimension 1 Anosov flows ${ }^{2}$ and uses some deeper results in foliation theory. The proof we shall present here has ingredients from both organized in a way that will lead us naturally to the generalization of these arguments to the classification problem of partially hyperbolic diffeomorphisms in dimension 3.

We emphasize the following fact. In dimension 2, the hairy ball theorem, or the Poincaré-Hopf index theorem, implies that admitting a continuous subbundle is already enough to get some topological obstruction (i.e., only the 2-torus and the Klein bottle admit a continuous splitting of the tangent bundle). However, this is not the case in dimension 3; up to double cover, every closed 3-manifold has trivial tangent bundle. That is, $T M \cong M \times \mathbb{R}^{3}$, therefore the existence of a splitting of the tangent bundle cannot be an obstruction by itself. It will be finer properties of the foliations that these bundles integrate, namely, the nonexistence of compact leaves, that will come in handy for this issue.

Remark 3.2. At the time that Margulis proved this theorem, the only known examples of Anosov flows in closed 3-manifolds were the geodesic flows in negative curvature (and its finite lifts), and the suspension flows of linear hyperbolic automorphisms of tori. Later, new examples started to appear, especially in dimension 3 (see [Bar]). The study of the geometry and topology of Anosov flows in dimension 3 has grown tremendously since these pioneering results. We refer the reader to [Bar] for a survey of the main results with several of the key ideas.

Remark 3.3. Theorem 3.1 implies the following result which also admits a more elementary proof just using the Lefschetz index. If $f: \mathbb{T}^{2} \rightarrow \mathbb{T}^{2}$ is an Anosov diffeomorphism $^{3}$ of a 2-torus, then the action of $f$ in homology is hyperbolic, meaning that it has no eigenvalue of modulus 1. An interesting challenge could be to prove this statement after (or before!) reading the proof below.

\section{The Proof}

We provide here a quick proof of Theorem 3.1 based on the original arguments, but probably with a more modern viewpoint. The goal is motivating tools that provide an understanding of the interaction between topology and dynamics.

An easy consequence of Theorem 3.1 is the nonexistence of Anosov flows in the sphere $S^{3}$. This can also be

\footnotetext{
$2_{i . e .,}$ those whose stable or unstable bundle is 1-dimensional

${ }^{3}$ An Anosov diffeomorphism $g: M \rightarrow M$ is such that Dg preserves a splitting $T M=E^{S} \oplus E^{u}$ so that vectors in $E^{S}$ are uniformly contracted and vectors in $E^{\mathcal{u}}$ are uniformly expanded as in $\$ 2$.
}

shown quite directly by a shortcut in the same argument: Assume that $\phi_{t}: S^{3} \rightarrow S^{3}$ is an Anosov flow. Consider $\mathcal{F}^{w s}$, the weak stable foliation of $\phi_{t}$ given by Theorem 2.1. By Novikov's compact leaf theorem (see Theorem 2.3) we know that every foliation by surfaces in $S^{3}$ must have a compact leaf; this contradicts Proposition 2.2.

With these elements in hand, we are ready to give the proof. The reader not comfortable with the basics of algebraic topology can use as a model the 3-torus $\mathbb{t}^{3}=$ $S^{1} \times S^{1} \times S^{1}=\mathbb{R}^{3} / \mathbb{Z}^{3}$ where integer translations are deck transformations of the universal cover $\mathbb{R}^{3}$. The theorem implies that $\mathbb{T}^{3}$ does not admit Anosov flows, and the difficulty of proving this case is the same as the general case. Here, one will have that $\widetilde{\mathbb{T}^{3}}=\mathbb{R}^{3}$ with the Euclidean metric (so balls do not have exponential growth of volume by a direct computation).

Proof of Theorem 3.1. Let $\pi: \tilde{M} \rightarrow M$ be the universal cover and lift $\phi_{t}$ to a flow $\tilde{\phi}_{t}: \widetilde{M} \rightarrow \widetilde{M}$. Let $\widetilde{\mathcal{F} w s}$ be the lift of the weak stable foliation to $\widetilde{M}$.

Consider an $\operatorname{arc} J$ tangent to the bundle $\tilde{E}^{u}$ (the lift of $\left.E^{u}\right)$. The arc $J$ is transverse to $\widetilde{\mathcal{F} w s}$. Since the foliation is invariant under $\tilde{\phi}_{t}$ and the arc $J$ maps to another arc tangent to $\tilde{E}^{u}$, we deduce that the $\operatorname{arc} \tilde{\phi}_{t}(J)$ cannot intersect the same foliation box twice. In particular, this would allow us to construct a transversal to $\mathcal{F}^{w s}$ which is homotopically trivial, contradicting Theorem 2.3 and Proposition 2.2. See Figure 4.

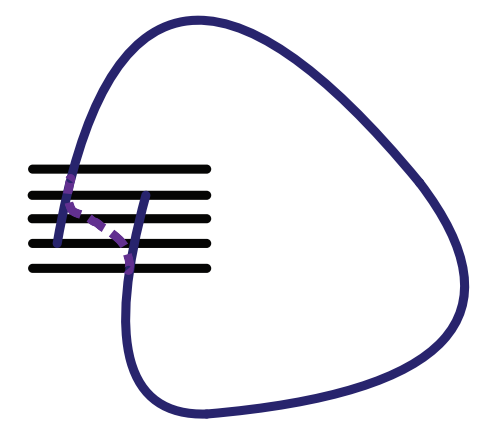

Figure 4. If a positively transverse curve intersects a leaf twice, one can construct a closed transversal.

Foliation boxes have uniform size since they can be pulled back from $M$ which is compact. One deduces that there exists a uniform constant $c_{0}>0$ so that

$$
\operatorname{vol}\left(B\left(\tilde{\phi}_{t}(J), 1\right)\right)>c_{0} \operatorname{length}\left(\tilde{\phi}_{t}(J)\right)
$$

where $B(X, r)$ denotes the set of points in $\tilde{M}$ with distance less than $r$ from $X$. Moreover, since $J$ is tangent to $E^{u}$ there are positive constants $c_{1}, \delta>0$ so that length $\left(\tilde{\phi}_{t}(J)\right)>$ $c_{1} e^{\delta t}$. Putting this together, one gets

$$
\operatorname{vol}\left(B\left(\tilde{\phi}_{t}(J), 1\right)\right)>c_{0} c_{1} e^{\delta t} .
$$


We will now show that there is a constant $c_{2}>0$ so that if $x_{0} \in J$, then $\tilde{\phi}_{t}(J)$ is contained in $B\left(x_{0}, R_{t}\right)$ where $R_{t} \leq c_{2} t+\operatorname{diam}(J)$. This is obtained by computing, for $x \in J$,

$$
d\left(x_{0}, \tilde{\phi}^{t}(x)\right) \leq d\left(x_{0}, x\right)+d\left(x, \tilde{\phi}_{t}(x)\right) \leq \operatorname{diam}(J)+c_{2} t,
$$

where $c_{2}$ is a bound on the norm for the vector field generating $\phi_{t}$.

This implies that $B\left(\tilde{\phi}_{t}(J), 1\right) \subset B\left(x_{0}, R_{t}+1\right)$ and therefore, taking $\hat{\delta}=\frac{\delta}{c_{2}}$ and $c_{3}=e^{-\hat{\delta}(\operatorname{diam}(J))}$, we get

$$
\operatorname{vol}\left(B\left(x_{0}, R_{t}+1\right)\right)>c_{0} c_{1} c_{3} e^{\hat{\delta}\left(R_{t}+1\right)},
$$

which gives (3.1) and completes the proof.

Margulis's proof is more elementary since it does not use any deep results about foliations; however, it depends crucially on the fact that the weak stable/unstable foliation is complete in the sense that a weak stable/unstable leaf is the union of the strong stable/unstable manifolds through points of a given orbit. This fact fails when one goes to the partially hyperbolic setting. This property is used by Margulis to construct by hand the universal cover of $M$ and compute its volume growth.

The proof of Plante and Thurston is similar to the one we present here; however, instead of computing volume they construct many loops that they show are pairwise non-homotopic. For this, they use Haefliger's argument (cf. footnote 1). In particular, as in the proof presented here, in contrast with Margulis's proof, it only needs one of the two foliations and hence it extends to codimension 1 Anosov flows. But what is important here is that this line of reasoning does not depend on understanding the internal structure of the codimension 1 foliation, and so it is well suited to be extended in other contexts.

\section{Classification of Partially Hyperbolic Systems}

We will now come to the problem of understanding the structure of general partially hyperbolic systems in 3dimensional manifolds by modelling the questions and ideas of the work done in the previous section. Here we shall concentrate on the following questions of current research interest which can be considered as continuations of the problem discussed above for Anosov flows:

Question 5.1. Which 3-manifolds admit partially hyperbolic diffeomorphisms? Which isotopy classes? Are these similar in some way to the known examples?

We refer the reader to [CP] for a general exposition of the basic facts about partially hyperbolic systems as well as a long list of examples. Here we will concentrate on a few relevant aspects specific to 3-dimensions.

A main difference which makes studying partially hyperbolic diffeomorphisms much harder than Anosov flows is that even if the strong bundles $E^{S}$ and $E^{u}$ still integrate uniquely into $f$-invariant foliations (essentially by the same argument as in the Anosov flow case), this is no longer true for the center stable and center unstable bundles $E^{c s}=E^{s} \oplus E^{c}$ nor $E^{c u}=E^{c} \oplus E^{u}$. This makes the study of partially hyperbolic diffeomorphisms much harder. At the beginning of its exploration, the topological study of these systems assumed the existence of such foliations under the concept of dynamical coherence since all the known examples had them. We say that a partially hyperbolic diffeomorphism is dynamically coherent if there are $f$ invariant foliations tangent respectively to $E^{c s}$ and $E^{c u}$.

A recent breakthrough result by Burago and Ivanov [BI] provided a tool for avoiding such an undesirable hypothesis. $^{4}$

Theorem 5.2. Up to finite cover, there is a Reebless foliation $\mathcal{F}$ transverse to the unstable direction $E^{u}$.

This implies by iterating backwards that one can choose the foliation to be as close to tangent to $E^{c s}$ as desired, but does not imply dynamical coherence, as in the limit the leaves could merge together forming what is called a branching foliation. This is an incredibly useful tool for the study of partially hyperbolic diffeomorphisms, but we do not discuss it here. We note here that the proof of Theorem 5.2 depends very strongly on the fact that $E^{c}$ and $E^{u}$ are 1-dimensional; indeed, one can not expect a similar result if $E^{c}$ has higher dimensions.

In fact, to show the result it is enough to show that there exists a foliation transverse to $E^{u}$ since the non-existence of Reeb components follows from the fact that there are no closed curves tangent to $E^{u}$. This just follows from the fact that a flow transverse to a Reeb component must have a closed orbit. This beautiful observation from [BI] allows them to treat the problem locally and obtain this global information.

Theorem 5.2 has the following consequence which is the first known topological obstruction for the existence of partially hyperbolic diffeomorphisms:

Corollary 5.3 (Burago-Ivanov). The sphere $\mathbb{S}^{3}$ does not admit partially hyperbolic diffeomorphisms.

The proof of Theorem 3.1 in $\$ 4$ has as a moral that to expand a 1-dimensional foliation transverse to a 2dimensional foliation in a 3-manifold one needs space. This moral extends to the diffeomorphism case, only diffeomorphisms can wrap the manifold onto itself and then obtain expansions without much space.

For instance, a matrix in $\operatorname{SL}(3, \mathbb{Z})$ with real eigenvalues, at least one of which is larger than 1 , induces a partially hyperbolic diffeomorphism on $\mathbb{T}^{3}$. The volume growth of

\footnotetext{
${ }^{4}$ The reason it is undesirable is that it is not easy to check, and several examples have appeared where it is known not to hold.
} 
the universal cover $\mathbb{R}^{3}$ of $\mathbb{T}^{3}$ is just polynomial. The reason is that the action of $f$ itself already gives the foliation space to expand. In the proof of Theorem 3.1 this appears in the crucial use of the fact that $\phi_{t}$ is a flow (or equivalently, that its time one map is homotopic to the identity) which gives equation (4.1).

With essentially the same proof as for Theorem 3.1 by replacing the stable manifold theorem with Theorem 5.2, one can obtain the following result which provides obstructions for the mapping classes which admit partially hyperbolic diffeomorphisms.

Theorem 5.4. If $f: M \rightarrow M$ is a partially hyperbolic diffeomorphism of a closed 3-dimensional manifold and $\hat{M}_{f}$ is the mapping torus of $f$, then the fundamental group $\pi_{1}\left(\hat{M}_{f}\right)$ of $\hat{M}_{f}$ has exponential growth.

Recall that the mapping torus of a map $F: X \rightarrow X$ is the space $X \times[0,1] / \sim$ where one identifies $(x, 1) \sim(F(x), 0)$ for all $x$. It depends only on the homotopy class of the map $F$, and produces a smooth manifold if $X$ is a manifold and $F$ a diffeomorphism (so that the equivalence with volume growth still holds).

But Theorem 5.2 is indeed stronger, since it can also provide further obstructions thanks to the well-developed theory of Reebless foliations. There are manifolds with exponential growth of fundamental group known not to admit foliations without compact leaves, including some hyperbolic 3-manifolds (see, e.g., [Ca, Example 4.4.6]). These also provide obstructions to the existence of partially hyperbolic diffeomorphisms. Up to recently, these were more or less all the known obstructions to the existence of partially hyperbolic diffeomorphisms. At the moment of this writing, we do not know any manifold with exponential growth of fundamental group which admits a partially hyperbolic diffeomorphism but does not admit an Anosov flow. But lots of developments have been made recently that give us hope that understanding partially hyperbolic diffeomorphisms is not far from understanding Anosov flows.

\section{Further Discussion}

As mentioned, the obstruction given by Theorem 5.4 is not sharp, so it makes sense to see to what extent one can characterize the homotopy classes of diffeomorphisms of 3-manifolds admitting partially hyperbolic diffeomorphisms. It turns out that only very recently examples in new isotopy classes were found [BGHP]. In these examples, new features of partially hyperbolic systems were exposed; in particular, the global nature of dynamical coherence is now better understood.

But somehow, all examples we know build in some way or the other on some Anosov system. The examples in [BGHP] are constructed by using the cone-field criterium to guarantee partial hyperbolicity together with a careful understanding of the global structure of the invariant bundles. This way, it is possible to construct diffeomorphisms of the manifold which respect transversalities between the bundles, and this allows us to create new partially hyperbolic diffeomorphisms in new isotopy classes. These kinds of constructions are still in their infancy, and it is likely that new examples can be created using these ideas. Nonetheless, there are some manifolds and isotopy classes of diffeomorphisms where the partially hyperbolic dynamics seem amenable to classification, notably hyperbolic and Seifert 3-manifolds [BFFP, BFP]. A notion of collapsed Anosov flow has been proposed recently that may account for all new examples, and which needs to be tested against new potential constructions [BFP].

In higher dimensions, Anosov systems are far from being classified, and new ways to construct partially hyperbolic examples have been devised [GHO], which depend to some extent on Anosov systems, but seem likely to be more flexible and may be combinable with techniques in [BGHP]. Even the most basic questions in high dimensions remain quite open.

We refer the reader to [BDV] for a general overview of smooth dynamics and to [Wil] for a recent account of partial hyperbolicity. In [CHHU] the reader can find a survey of the dynamics of partially hyperbolic diffeomorphisms specialized to dimension 3, which also touches upon the classification problem.

If the reader wishes to know more about the classification problem of partially hyperbolic diffeomorphisms in dimension 3, then the following references could provide a useful introduction [CHHU, HP, Pot, BFFP, BFP].

ACKNOWLEDGMENTS. The author was partially supported by CSIC-618, FCE-135352, the Minerva Research Foundation Membership Fund, and the grant NSF DMS-1638352. This work was written while the author was a von Neumann Fellow at the Institute for Advanced Study, and he wants to acknowledge the excellent working conditions and environment. Comments of Silvia Ghinassi, Mariana Haim, Santiago Martinchich, Martín Reiris, and Jan Vonk were very helpful in the writing of the note. The author wishes to particularly thank the referees who provided a lot of helpful input to improve the paper.

\section{References}

[Ano] D. V. Anosov, Geodesic flows on closed Riemannian manifolds of negative curvature (Russian), Trudy Mat. Inst. Steklov. 90 (1967), 209. MR0224110

[AnS] D. V. Anosov and Ja. G. Sinaĭ, Certain smooth ergodic systems (Russian), Uspehi Mat. Nauk 22 (1967), no. 5 (137), 107-172. MR0224771 
[Bar] T. Barthelme, Anosov flows in 3-manifolds (2017), notes available on the author's webpage.

[BFFP] T. Barthelme, S. Fenley, S. Frankel, and R. Potrie, Partially hyperbolic diffeomorphisms homotopic to the identity in dimension 3, arXiv:1908.06227.

[BFP] T. Barthelme, S. Fenley, and R. Potrie, Collapsed Anosov flows and self orbit equivalences, preprint (2020).

[BDV] C. Bonatti, L. J. Díaz, and M. Viana, Dynamics beyond uniform hyperbolicity: A global geometric and probabilistic perspective, Encyclopaedia of Mathematical Sciences, Mathematical Physics, III, vol. 102, Springer-Verlag, Berlin, 2005. MR2105774

[BGHP] C. Bonatti, A. Gogolev, A. Hammerlindl, and R. Potrie, Anomalous partially hyperbolic diffeomorphisms III: abundance and incoherence, Geom. Topol. 24 (2020), no. 4, 1751-1790, DOI 10.2140/gt.2020.24.1751. MR4173921

[BI] D. Burago and S. Ivanov, Partially hyperbolic diffeomorphisms of 3-manifolds with abelian fundamental groups, J. Mod. Dyn. 2 (2008), no. 4, 541-580, DOI 10.3934/jmd.2008.2.541. MR2449138

[Ca] D. Calegari, Foliations and the geometry of 3-manifolds, Oxford Mathematical Monographs, Oxford University Press, Oxford, 2007. MR2327361

[CHHU] P. D. Carrasco, F. Rodriguez-Hertz, J. RodriguezHertz, and R. Ures, Partially hyperbolic dynamics in dimension three, Ergodic Theory Dynam. Systems 38 (2018), no. 8, 2801-2837, DOI 10.1017/etds.2016.142. MR3868015

[CP] S. Crovisier and R. Potrie, Introduction to partially hyperbolic dynamics (2015), notes available on the author's webpage.

[Do] C. I. Doering, Persistently transitive vector fields on threedimensional manifolds, Dynamical systems and bifurcation theory (Rio de Janeiro, 1985), Pitman Res. Notes Math. Ser., vol. 160, Longman Sci. Tech., Harlow, 1987, pp. 5989. MR907891

[GHO] A. Gogolev, P. Ontaneda, and F. R. Hertz, New partially hyperbolic dynamical systems I, Acta Math. 215 (2015), no. 2, 363-393, DOI 10.1007/s11511-016-0135-3. MR3455236

[HP] A. Hammerlindl and R. Potrie, Partial hyperbolicity and classification: a survey, Ergodic Theory Dynam. Systems 38 (2018), no. 2, 401-443, DOI 10.1017/etds.2016.50. MR3774827

[Mar] G. Margulis, Y-flows on three dimensional manifolds; Appendix to Certain smooth ergodic systems, Uspehi Mat. Nauk 22 (1967), no. 5, 107-172, by D. Anosov and Y. Sinai.

[PT] J. F. Plante and W. P. Thurston, Anosov flows and the fundamental group, Topology 11 (1972), 147-150, DOI 10.1016/0040-9383(72)90002-X. MR295389

[Pot] R. Potrie, Robust dynamics, invariant structures and topological classification, Proceedings of the International Congress of Mathematicians-Rio de Janeiro 2018. Vol. III. Invited lectures, World Sci. Publ., Hackensack, NJ, 2018, pp. 20632085. MR3966842

[Wil] A. Wilkinson, Mechanisms for chaos (2019), notes available on the author's webpage.

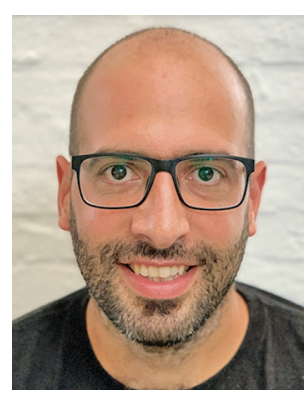

Rafael Potrie

Credits

Opener photo is courtesy of Free art director via Getty.

Figures 1-4 are courtesy of Rafael Potrie.

Author photo is courtesy of Natalia de León.

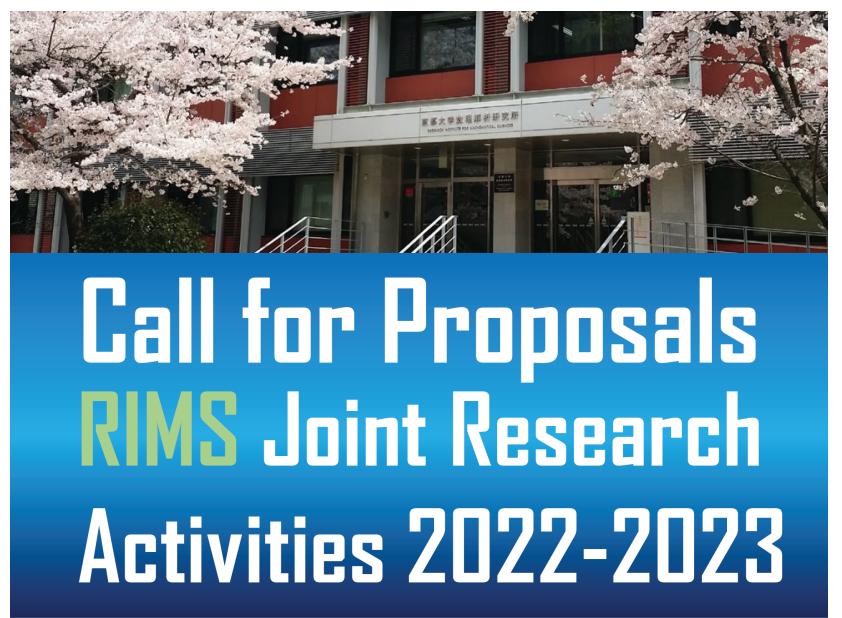

Application deadline : August 31, 2021, 23:59 (JST)

Types of Jaint Research Activities More Information :

*RIMS Satellite seminars 2022 RIMS Int.Jل/RC Website

*RIMS Review seminars 2022 http://www.kurims.kyoto

*RIMS Warkshops Type C 2022 -u.ac.jp/kyoten/en/

*RIMS Research Project 2023

京都大学

KYOTO UNIVERSITY
$\boldsymbol{R} I \mathbf{M S}$ Research Institute for 